\title{
Preoperative Anesthesiology Consult Utilization in Ontario - A Population-Based Study
}

\author{
Joanna Dion ${ }^{1}$, Robert Campbell ${ }^{1}$, Paul Nguyen ${ }^{1}$, and Jason Beyea ${ }^{1}$ \\ ${ }^{1}$ Queen's University
}

October 6, 2020

\begin{abstract}
ABSTRACT $\neg$ Rationale, aims and objectives: Physician consultations are a limited resource. Anesthesiologists provide anesthesia during surgery and procedures, prepare patients for surgery in preoperative clinics, and provide postoperative care. This study sought to evaluate current consultation usage patterns, with an aim to determine possible opportunities for efficiency. Method: A retrospective comprehensive population-based cohort study was performed, evaluating all hospitals in the Canadian province of Ontario from 2002-2018. The main outcome measures were American Society of Anesthesiologists (ASA) classification of the patients, and whether the patients underwent surgery within 3 months following the anesthesia consultation. Results: A cohort of 2,023,499 patients, and a total of 2,920,100 preoperative anesthesia consultations was obtained. The number of consults per year doubled between 2003 (112,983/year) and 2017 (246,427/year). Each year, an average of $19.32 \%$ of the consults (range: 17.69-20.49\%) were for patients that did not progress to having surgery. Of those that did have surgery following the anesthesia consult, $37.23 \%$ were ASA Classification I or II. The most common surgical procedures (percent of total) following anesthesia consult were: Knee implantation of internal device (9.46\%), hip implantation of internal device $(5.84 \%)$, cataract excision $(4.09 \%)$, repair of muscle of chest/abdomen $(3.31 \%)$, uterus excision (2.76\%), and gallbladder excision $(2.67 \%)$. Conclusions: This study reveals data on utilization and trends over time of preoperative anesthesia consultations. Potential opportunities for optimization were found, including patients who did not proceed to surgery, and healthier patients undergoing low to moderate risk surgery that may not require consultation.
\end{abstract}

\section{Main Text \\ Introduction}

Recently, increasing attention has been focused on efficient utilization of healthcare services. Approximately 1.2 million outpatient and 350,000 inpatient surgeries were performed in Ontario in 2014/2015 ${ }^{1,2}$. Many patients undergo preoperative screening in the form of laboratory or imaging investigations or consultation with an anesthesiologist. This process of investigations and consultation optimizes patients medically by identifying and improving unstable conditions which might result in less perioperative complications ${ }^{3}$.

As perioperative physicians, anesthesiologists provide significant guidance to help determine which patients should undergo testing before elective surgery. Since 2014, the Choosing Wisely Canada campaign has endeavoured to help determine appropriate and necessary treatment by medical specialty ${ }^{4}$. The Choosing Wisely Canada recommendations for anesthesia include the avoidance of laboratory or other cardiovascular testing for asymptomatic patients undergoing lower risk surgeries. Several studies assessing the use of investigations prior to low risk surgery showed that many patients continue to undergo potentially unnecessary testing ${ }^{5,6}$.

There are currently no guidelines recommending appropriate use of anesthesia consultation, and there appears to be significant practice variation ${ }^{3,7}$. Wijeysundera et. al. ${ }^{8}$ studied the use of anesthesia consultation in and found an increase in preoperative anesthesia consultations from $19 \%$ in 1994 to $53 \%$ in 2003 . Patients who received anesthesia consultations had lower risks of anxiety and day of surgery cancellations, 
as well as slightly shorter hospital stays. Mortality risks were not different, and questions regarding the cost-effectiveness of preoperative consultations were raised. Overall, there appears to be a trend towards increasing use of anesthesia consultation, even amongst patients undergoing low risk surgeries, which potentially could result in inefficient use of health care resources ${ }^{9}$. Presumably, higher risk patients undergoing more invasive surgeries will get the most benefit from preoperative consultation ${ }^{3,7}$. Similarly, there are no specific guidelines for which patients can benefit from an internal medicine consultation prior to surgery ${ }^{10}$.

In the current climate of limited health care resources, the authors sought to obtain information regarding current and historical usage of preoperative anesthesia consults. Herein, we carried out a population-based study to evaluate the use of preoperative anesthesia consultation services.

\section{Methods}

\section{Overview, study population and study design}

We carried out a population-based study to evaluate usage of preoperative anesthesia consultation in . is the largest province in, with a population of over 14 million residents. The Canadian healthcare system is a publically funded and administered system, governed by the Canada Health Act. This Act mandates comprehensive universal coverage for all medically necessary services, including medical (anesthesiology) consultations. Private health insurance for these services is prohibited. As such, these consults are available to all persons equally, and the data sources were population-based. Patients were excluded if a valid patient identifier was not available, if age/sex information was missing, or if residency was outside of . This study was approved by the Queen's University Health Sciences \& Affiliated Teaching Hospitals Research Ethics Board, project \#6025862.

\section{Data Sources and Data Linkage}

Administrative datasets housed at the ICES were used to obtain the data for this study. ICES is an independent, non-profit research organization funded by the Ontario Ministry of Health and Long Term Care whose data holdings include all health care related events for the population of Ontario. The Registered Persons Database (RPDB) is a repository of demographic information for all residents of who are eligible for the Ontario Health Insurance Plan (OHIP). RPDB is maintained by the Ministry, and contains information on health card number, date of birth, sex, address, and deceased date (where applicable). Personal identifying information in RPDB is removed at ICES, and each unique health number is converted into an anonymous unique identifier, the ICES Key Number (IKN). The IKN is a common identifier that is used to link data sources within ICES. The OHIP database contains data on the diagnosis and fee-for-service claims submitted by physicians and paid by the universal health care system. The Canadian Institute for Health Information Discharge Abstract Database and Same Day Surgery database contain information on all inpatient and day surgery visits, respectively, abstracted from hospital records for patient diagnoses and procedures.

The datasets were created at ICES Queen's based on patients of all ages, using physician OHIP billing for an outpatient anesthesia consult (OHIP fee code: A015, A016) (Table 1) from April 1, 2002 to June 30, 2018, and hospital discharge records from April 1, 2002 to September 30, 2018 (study period). Patients were excluded if they had missing age/sex data, or were a resident of another province at the time of consultation or surgery. These datasets were then linked to demographic data, and to hospital inpatient and outpatient discharge data for surgeries.

The study cohort included all patients who underwent an outpatient preoperative anesthesia consultation (A015, A016) during the study period. The index date was the first use of these OHIP billing claims. This cohort permitted reporting of counts and percentages of surgery, and for those patients who underwent surgery, the ASA classification (American Society of Anesthesiologists physical status classification system) of the patients by the anesthesiologist at the time of surgery. The ASA classification is used ubiquitously to stratify the general health of patients whereby an ASA I patient is healthy with no medical diagnoses, all the way up to an ASA V patient who is so medically complex that he or she is not expected to survive for 24 hours with or without surgical intervention (Table 2) ${ }^{11}$. For ASA III, IV, and V patients, there 
are respective anesthesia modifier billing codes through OHIP. This is due to the increased complexity of providing perioperative anesthesia care for patients with increased comorbidities. There are no modifier ASA billing codes for patients who are deemed ASA I or ASA II (healthier) at the time of surgery by the attending anesthesiologist. As such, if a modifier for ASA III, IV, or V was not used, the patient was presumed to be ASA I or II.

\section{Outcomes and Analyses}

This study sought to determine the volume of consults, and to determine if the patient progressed to surgery. Also, for those patients who had surgery, the ASA classification was determined. Cohorts including usage of preoperative anesthesia consults over time, those who went on to have surgery (specifically, the most significant surgical intervention during the inpatient or day surgery visit), and ASA classification as assigned at the time of surgery were created. Frequencies and percentages of preoperative anesthesia consults, most common types of surgery and ASA classifications were quantified. Modified Poisson regression analyses were used to provide the relative risks of consults for the most common types of surgery and place of preoperative anesthesia consults. To account for the patient-level clusters of repeated surgeries over time, the Poisson regression models used the generalized estimating equations (GEE) approach.

Descriptive statistics and regression analyses were generated using the SAS Enterprise Guide software version 7.1 (SAS Institute, Cary, NC) at ICES.

\section{Results}

\section{Cohort characteristics}

Over the study period, 2,920,100 preoperative anesthesiology consults were performed in Ontario on 2,023,499 patients. The average patient age was $59.02 \pm 19.28$ years (range $1-108$ years), and $46.43 \%$ were male. The annual volume of these consults doubled over the study period (Figure 1). Of patients who proceeded to have surgery within the 3 months after the anesthesiology consult, $97.58 \%$ of the consults were performed electively in the outpatient anesthesiology clinic setting.

\section{Surgery}

Of the anesthesiology consults performed in Ontario, an average of 19.32\% (range: 17.69-20.49\%) of these consults were for patients who did not proceed to surgery in the ensuing three months. Expanding the postconsult window, averages of $18.58 \%$ (ensuing 4 months), $17.67 \%$ (ensuing 6 months), and $16.15 \%$ (ensuing 12 months) did not proceed to surgery. Looking specifically at the ensuing three month window, the results were stable over time (Figure 2). Of patients who proceeded to surgery, joint replacement and cataract surgeries were the most common procedures (Table 3 ).

\section{American Society of Anesthesiologists (ASA) Classification of Surgical Patients}

The ASA classification of the patients was assigned at the time of surgery by the attending anesthesiologist, through the respective billing codes. Analysis of these codes revealed that over $37 \%$ of patients were classified as either ASA I or ASA II (Figure 3). However, the percentage of patients receiving preoperative anesthesiology consults who were ASA I or ASA II declined over the study period (Figure 4). ASA class I or II consults were slightly more likely to be ordered by surgeons under age 36 and over age 60 (Figure 5). Patients undergoing cataract surgery had a higher likelihood of receiving an anesthesia consultation if they had an ASA I or II, whereas knee implant, chest/abdominal muscle repair, bladder excision, and coronary artery bypass were more likely to have a consult if they were ASA III-V (Table 3). Furthermore, there was notable variability in consults by geographical region. In Ontario, healthcare geographical regions have traditionally been divided into fourteen Local Health Integration Networks (LHIN). In 2003, the proportion of consultations that were ASA I/II ranged by LHIN from $38.6 \%$ to $68.8 \%$ of all preoperative anesthesia consults. In 2017, this proportion ranged by LHIN from $20.2 \%$ to $33.2 \%$ of all preoperative anesthesia consults.

\section{Discussion}


As health care costs continue to rise in Canada $^{12}$, more emphasis has been directed towards efficient usage of resources. The first step is to evaluate the current landscape. As physicians, there is an opportunity to address the health care system based on medical necessity, and to look for efficiencies. This study evaluated a large, comprehensive cohort from a single-payer healthcare system, and identified current and historical anesthesia consult utilization.

Individual centres have demonstrated that preoperative anesthesia consultations reduce patient anxiety ${ }^{13}$, reduce cancellations on the day of surgery ${ }^{14-16}$, and reduce hospital $\operatorname{costs}^{17}$. It is unclear though whether these same benefits are borne out when applied to a population.

The Canadian Institute for Health Information reports that $15.1 \%$ of health care expenditures are paid for physician services ${ }^{18}$. In addition to the physician costs, there are costs for facility/infrastructure, additional personal (including nurses, receptionists, custodial staff), laboratory testing, and medical imaging. Furthermore, it has been reported that preoperative anesthesia consultations result in higher rates of ordering possibly unnecessary specialized testing, including echocardiography ${ }^{8}$. It is important to note that echocardiography provides minimal additional prognostic information above clinical risk factors ${ }^{19}$ and additional bloodwork, for example Brain Natriuretic Peptide (BNP $)^{20}$.

A major concern from a surgical and health systems/resource management standpoint is the possibility of cancellation on the day of surgery. As well, the authors recognize that system and cultural differences exist at different hospitals, and by different physicians, in terms of utilization of these preoperative consultations. This is supported by the present study, which described geographic variability in the usage of preoperative anesthesiology consultations. However, the worry of surgery cancellation may feed, at least in part, increased usage of preoperative anesthesia consultations. It may be possible that through the use of structured medical directives and identification of patients in need of consultation by the hospital's presurgical screening clinic, some fears can be mitigated. The authors acknowledge that this is a much-needed area of research.

An interesting subgroup identified consisted of patients undergoing preoperative anesthesia consults prior to cataract surgery. Thilen et al. ${ }^{9}$ found a substantial increase from 1995 to 2006 in preoperative consultation prior to cataract surgery. The current study found that after knee and hip replacement, the third most common operation leading to utilization of preoperative anesthesia consultation was cataract surgery. This may be due in part to older and more medically complex patients being offered cataract surgery than in the past. Also, it may be that additional procedures are being completed at the same time to address glaucoma or other concomitant issues. However, it is also possible that policies and routine approaches in some centres might find efficiencies by re-evaluating the need for such consultations.

It is interesting that the percentage of ASA I and II patients who receive preoperative anesthesia consultations has decreased markedly over time (Figure 4). This may already reflect the strains and limitations being placed on the healthcare system. There is a slight tendency for early and late career surgeons to order more ASA I/II preoperative consults compared to mid-career physicians (Figure 5). At this time, it is not clear why this pattern was observed, however, the findings are consistent with previous research showing a tendency for younger physicians to order more preoperative investigations ${ }^{21}$. As our system responds to the pressures of increasing numbers and comorbidities of patients, likely many hospitals are already finding efficiencies. Also, the movement towards limiting unnecessary preoperative testing (Choosing Wisely Canada) is likely taking hold. The results of this study provide data to further the discussion surrounding preoperative anesthesia consultations.

There are limitations that are intrinsic to administrative data. There are many good reasons why some ASA I and II patients should undergo preoperative anesthesia consultation that may not appear in the datasets used. For example, patient factors such as features predicting very difficult airway management, language barrier, extreme anxiety surrounding anesthesia or a personal or family history of problematic anesthesia would be good indications for anesthesia consultation. These factors might not be captured by the ASA classification in terms of ASA III or above. Similarly, longer or more complex surgeries requiring management including prone positioning, one-lung ventilation, or the anticipation of significant blood loss 
or fluid shifts or potential for significant postoperative pain would be reasons for anesthesia consultation in a healthy patient. Having said this, these sorts of surgeries were not in the top five most common procedures captured by this study.

Data quality also relies on initial accurate coding by the physician and hospital coders. A comparison of administrative data with hospital chart data concluded that major events (surgical procedures, mortality, patient demographics, primary diagnoses) are accurately coded ${ }^{22}$.

An interesting group of patients are those 19.3\% who underwent an anesthesia consultation and then did not proceed to surgery within 3 months. There are a few possible explanations for this. First, it may be that after discussion with the anesthesiologist the patient decided not to go ahead with the surgical procedure. This can occur after an individualized explanation of medical risks or postoperative predicted morbidity or mortality based on patient and surgical factors. For example, a medically complex patient may choose not to undergo a hip replacement to avoid a potential postoperative complication. This is a great use of the system and allowed patient autonomy and informed shared decision making. Secondly, it may be that the surgery was postponed outside the 3 month window due to medical or scheduling reasons. This patient would then likely undergo surgery at a later time (after potentially undergoing further pre-operative assessment). This is a reality of our medical system, and offers opportunities to ensure we repeat as little as medically necessary to get the patient ready for the delayed surgery. Finally, after undergoing anesthesia consultation, the patient may not undergo surgery because it is no longer required, or the patient changes his or her mind - unrelated to the anesthesiologist assessment. Further understanding this group of patients could potentially provide some areas for increasing efficiency.

The results demonstrated a doubling of anesthesia consults per year over the course of the study. For a meaningful understanding of this number, the denominator (number of surgeries per year) is needed. The authors acknowledge that this information is not straightforward to obtain in a reliable manner. To the best of our knowledge, the data from Statistics Canada would be the most applicable. Evaluation of Statistics Canada waiting times for non-emergency surgery ${ }^{23}$ revealed that in Ontario, yearly surgical volumes increased modestly from 544,002 in 2005 to 704,890 in 2013, an increase of $29.6 \%$. The data from the present study indicates a much larger increase for anesthesia consults of $92.9 \%$ (from 112,983 to 217,959) over this timeframe (2005-2013). This may be due to many factors, including increased numbers of patient medical comorbidities, increased surgical procedure complexity, surgeon/anesthesiologist/hospital preference, and patient desire for discussion surrounding the anesthetic itself.

\section{Conclusion}

This study reveals important data relating to the utilization and trends over time of preoperative anesthesia consultations within a large comprehensive population. Opportunities for discussion were found, including consultations provided for patients who did not proceed to surgery, and those healthier patients undergoing minimally to moderately invasive surgeries. These findings are consistent with the Choosing Wisely Canada section for anesthesia which suggests minimizing preoperative testing for asymptomatic patients (synonymous with ASA class I and II patients) undergoing minimally invasive surgeries. Future studies are needed to evaluate the safety of allocating preoperative anesthesiology consultations in particular groups, and the potential healthcare system efficiencies and economic benefits of these reductions.

\section{References}

1. Canadian Institute for Health Information, DAD/HMDB Inpatient Hospitalizations: Volumes, Length of Stay, and Standardized Rates, QuickStats.

2. Ontario Hospital Association, Quick Facts 2016: Ontario's Public Hospitals. Available from: https://www.oha.com/guidance-and-resources/hospital-physician-relations

3. Wijeysundera DN. Preoperative consultations by anesthesiologists. Curr Opin Anaesthesiol. 2011 Jun;24:326-30. 
4. Levinson W, Huynh T. Engaging physicians and patients in conversations about unnecessary tests and procedures: Choosing Wisely Canada. CMAJ. 2014;186:325-6.

5. Kirkham KR, Wijeysundera DN, Pendrith C, Ng R, Tu JV, Laupacis A, Schull MJ, Levinson W, Bhatia RS. Preoperative testing before low-risk surgical procedures. CMAJ. 2015;187:E349-E358.

6. Kirkham KR, Wijeysundera DN, Pendrith C, Ng R, Tu JV, Boozary AS, Tepper J, Schull MJ, Levinson W, Bhatia RS. Preoperative Laboratory Investigations: Rates and Variability Prior to Low-risk Surgical Procedures. Anesthesiology. 2016;124:804-14.

7. Thilen SR, Wijeysundera DN, Treggiari MM. Preoperative Consultations. Anesthesiol Clin. 2016;34:17-33.

8. Wijeysundera DN, Austin PC, Beattie WS, Hux JE, Laupacis A. A population-based study of anesthesia consultation before major noncardiac surgery. Arch Intern Med. 2009;169:595-602.

9. Thilen SR, Treggiari MM, Lange JM, Lowy E, Weaver EM, Wijeysundera DN.

Preoperative consultations for medicare patients undergoing cataract surgery. JAMA Intern Med. 2014;174:380-8.

10. Wijeysundera DN, Austin PC, Beattie WS, Hux JE, Laupacis A. Variation in the practice of preoperative medical consultation for major elective noncardiac surgery: a population-based study. Anesthesiology. 2012;116:25-34.

11. Abouleish AE, Leib ML, Cohen NH. ASA provides examples to each ASA physical status class. ASA Monitor 2015; 79:38-9 http://monitor.pubs.asahq.org/article.aspx?articleid=2434536

12. https://www.cihi.ca/en/health-spending/2018/national-health-expenditure-trends/how-has-healthspending-growth-changed-over-the-last-40-years

13. Klopfenstein CE, Forster A, Van Gessel E. Anesthetic assessment in an outpatient consultation clinic reduces preoperative anxiety. Can J Anaesth. 2000;47:511-5.

14. van Klei WA, Moons KG, Rutten CL, Schuurhuis A, Knape JT, Kalkman CJ, Grobbee DE. The effect of outpatient preoperative evaluation of hospital inpatients on cancellation of surgery and length of hospital stay. Anesth Analg. 2002;94:644-9.

15. Ferschl MB, Tung A, Sweitzer B, Huo D, Glick DB. Preoperative clinic visits reduce operating room cancellations and delays. Anesthesiology. 2005;103:855-9.

16. Farasatkish R, Aghdaii N, Azarfarin R, Yazdanian F. Can preoperative anesthesia consultation clinic help to reduce operating room cancellation rate of cardiac surgery on the day of surgery? Middle East J Anaesthesiol. 2009;20:93-6.

17. Pollard JB, Garnerin P, Dalman RL. Use of outpatient preoperative evaluation to decrease length of stay for vascular surgery. Anesth Analg. 1997;85:1307-11.

18. https://www.cihi.ca/en/health-spending/2018/national-health-expenditure-trends/where-is-most-ofthe-money-being-spent-in-health-care-in-2018

19. Halm EA, Browner WS, Tubau JF, Tateo IM, Mangano DT. Echocardiography for assessing cardiac risk in patients having noncardiac surgery. Study of Perioperative Ischemia Research Group. Ann Intern Med. 1996;125:433-41.

20. Duceppe E, Parlow J, MacDonald P, Lyons K, McMullen M, et. al. Canadian Cardiovascular Society Guidelines on Perioperative Cardiac Risk Assessment and Management for Patients Who Undergo Noncardiac Surgery. Canadian Journal of Cardiology. 2017;33(1):17-32. 
21. Beyea JA, Rosen E, Stephens T, Nguyen P, Hall SF. Audiometric Testing Guideline Adherence in Children Undergoing Tympanostomy Tubes: A Population-Based Study. Otolaryngol Head Neck Surg. 2018;158:11271133.

22. Hawker GA, Coyte PC, Wright JG, Paul JE, Bombardier C. Accuracy of administrative data for assessing outcomes after knee replacement surgery. J Clin Epidemiol. 1997;50:265-73.

23. Statistics Canada, Data, Non-emergency surgeries, by waiting times (https://www150.statcan.gc.ca/t1/tbl1/en/tv.action?pid=1310048201\&pickMembers\%5B0\%5D=1.7). Accessed June 22, 2020.

\section{Acknowledgements}

1. This study was supported by ICES, which is funded by an annual grant from the MOHLTC. The opinions, results and conclusions reported in this paper are those of the authors and are independent of the funding sources. No endorsements by ICES or the Ontario MOHLTC is intended or should be inferred.

2. The dataset was created at ICES Queen's (Kingston, Ontario). The dataset creation and data analysis were performed at ICES Queen's by Dr. Paul Nguyen.

3. Parts of this material are based on data and/or information compiled and provided by CIHI. However, the analyses, conclusions, opinions and statements expressed in the material are those of the author(s), and not necessarily those of CIHI.

\section{Conflict of Interest}

Role of Funders: Financial support only.

Conflict of Interest: No conflicting relationships exist for any author.

Financial Disclosure: No financial interests exist for any author.

Tables

Table 1. Ontario Health Insurance Plan (OHIP) administrative billing codes

\begin{tabular}{ll}
\hline Description of Code & Billing Code \\
\hline Anesthesia consult, repeat consult & A015, A016 \\
ASA III modifier & E022C \\
ASA IV modifier & E017C \\
ASA V modifier & E016C \\
\hline
\end{tabular}

ASA: American Society of Anesthesiologists Physical Status Classification System

Table 2. American Society of Anesthesiologists (ASA) Physical Status Classification System (Abouleish et al.2015) $)^{11}$

\begin{tabular}{lll}
\hline ASA Classification & Definition & Adult Example \\
\hline ASA I & A normal healthy patient & Healthy non-smoker \\
ASA II & Mild systemic disease & Well-controlled hypertension \\
ASA III & Severe systemic disease & Poorly controlled diabetes \\
ASA IV & Severe systemic disease that is a constant threat to life & Myocardial infarction within th \\
ASA V & Moribund patient, not expected to survive without the operation & Intracranial bleed with mass eff \\
\hline
\end{tabular}

Table 3. Most common surgical procedures of patients who underwent a preoperative anesthesiology con- 
sultation

\begin{tabular}{lll}
\hline Procedure & Percent of Total Procedures & Relative Risk* $(\mathbf{9 5 \%}$ CI) of AS \\
\hline Knee, implantation of internal device & 9.46 & $0.711(0.537-0.942)$ \\
Hip, implantation of internal device & 5.84 & $0.810(0.613-1.072)$ \\
Cataract excision & 4.09 & $1.364(1.025-1.814)$ \\
Repair, muscle of chest \& abdomen open approach & 3.31 & $0.745(0.567-0.979)$ \\
Excision total, uterus & 2.76 & $1.109(0.838-1.466)$ \\
Excision total, gallbladder & 2.67 & $0.879(0.667-1.157)$ \\
Repair, knee joint arthroscopic approach & 1.95 & $0.999(0.760-1.313)$ \\
Excision partial, bladder & 1.80 & $0.296(0.083-1.059)$ \\
Coronary arteries bypass, open approach & 1.68 & $0.055(0.042-0.071)$ \\
Cesarean section & 1.62 & $1.300(0.983-1.721)$ \\
\hline
\end{tabular}

CI: Confidence interval

* Relative risks computed from modified Poisson regression models for clustered data

\section{Figure Legends}

Figure 1. Preoperative anesthesiology consultations per year in Ontario (2003-2017).

Figure 2. Outpatient anesthesiology consultations for patients who did not proceed to a surgical procedure in the subsequent three months.

Figure 3. Distribution of patients by ASA classification. ASA class V represents $<0.50 \%$ of patients.

Figure 4. Percentage per year of patients who received preoperative anesthesia consultation and were classified as ASA I or II at the time of surgery.

Figure 5. Relative risk (with $95 \%$ confidence limit bands) of ASA I or II class patient (vs. ASA III-V) preoperative anesthesia consultation versus surgeon age. Age 46-50 surgeon group is the reference group.

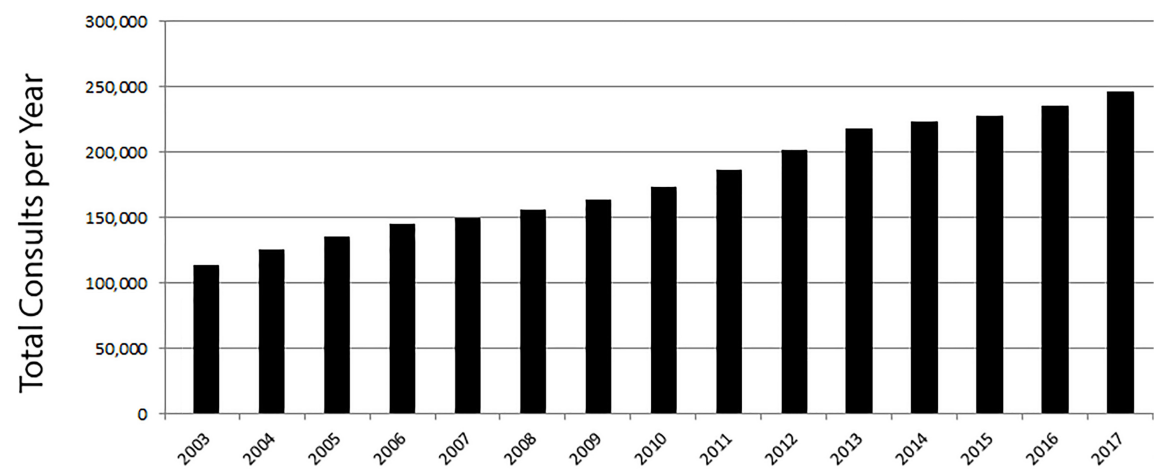



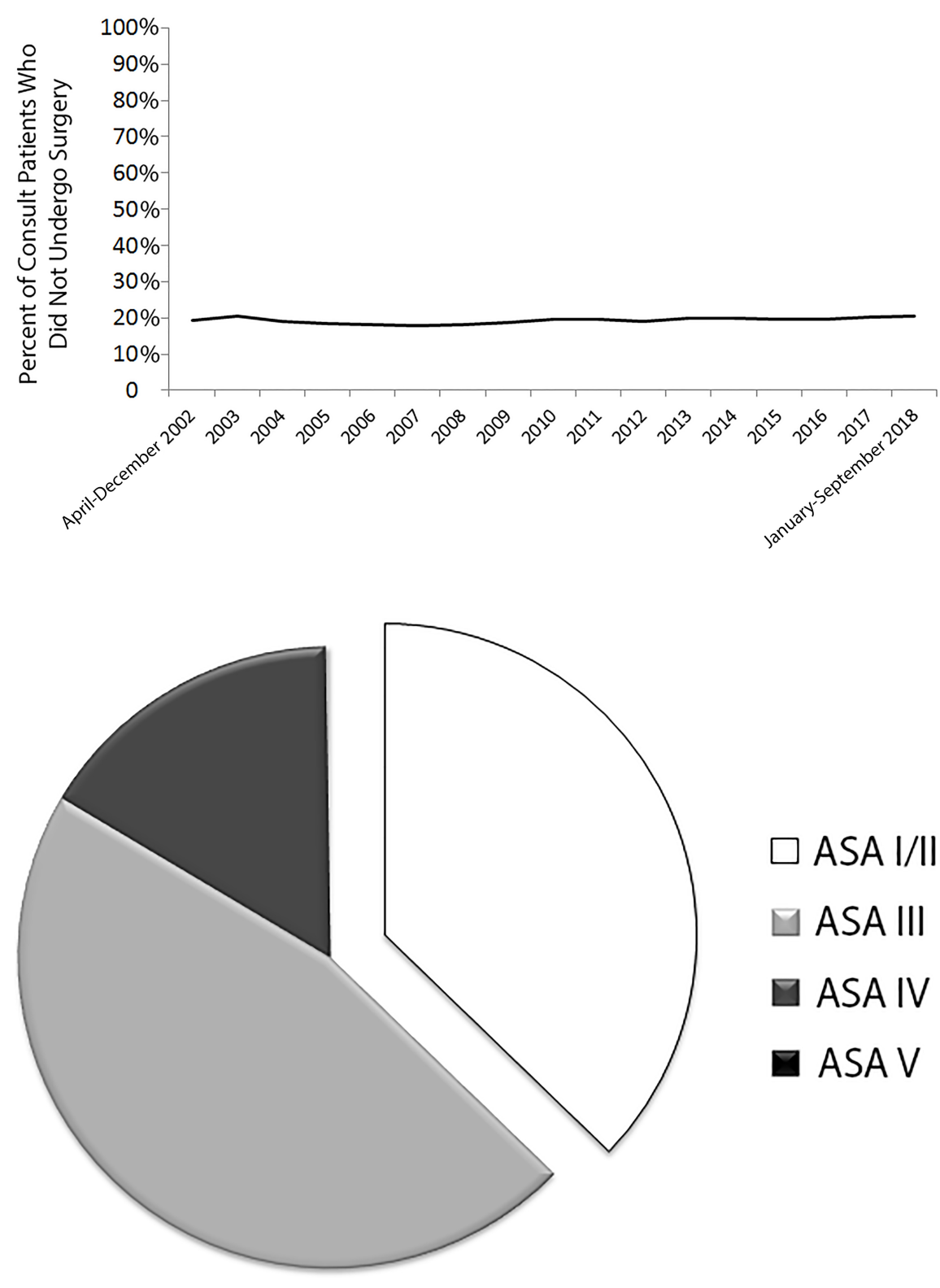

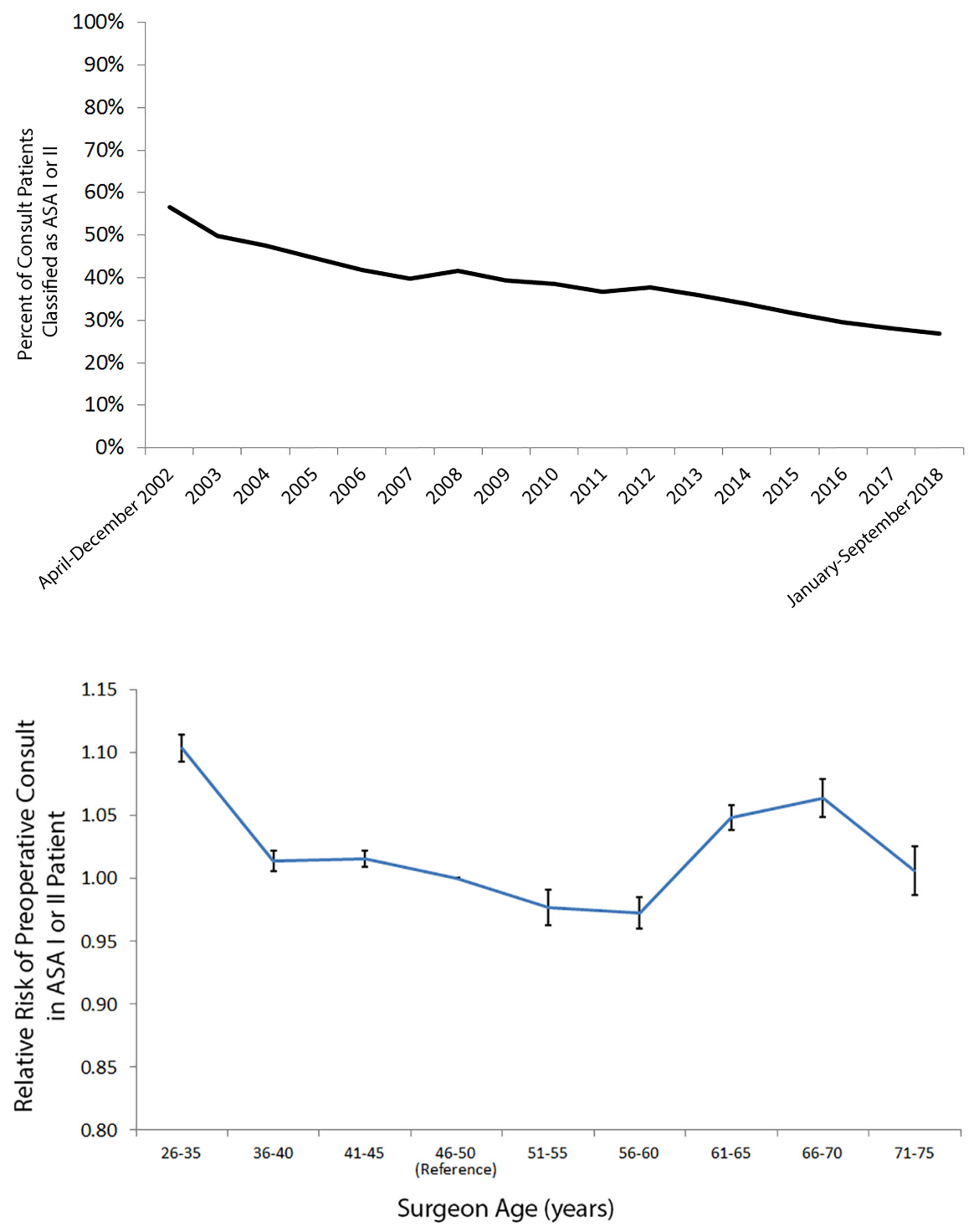